\title{
UBIBOT: UM AGENTE CONVERSACIONAL CIENTE DO CONTEXTO DE APRENDIZAGEM DO USUÁRIO
}

\author{
Leo Natan Paschoal, CCHS/UNICRUZ, leonatanpaschoal@gmail.com \\ Patricia M. Mozzaquatro Chicon, CCHS/UNICRUZ, patriciamozzaquatro@ gmail.com \\ Gilse Antoninha Morgental Falkembach, CINTED/UFRGS, gilsemf@ gmail.com
}

\begin{abstract}
Resumo: Este artigo apresenta uma ferramenta computacional que integra os conceitos de aprendizagem ubíqua, a fim de contribuir para o processo de ensino e aprendizagem. Nesse sentido, descreve sobre o desenvolvimento de um agente conversacional, integrado no ambiente virtual de aprendizagem Moodle, que objetiva prover suporte ao ensino da disciplina de Engenharia de Software. O agente, intitulado Ubibot, é capaz de identificar os contextos dos estudantes e responder as dúvidas dos mesmos. Nesta pesquisa, os contextos são representados pelo nível de conhecimento e desempenho do estudante. Além disso, o Ubibot utiliza tecnologia web responsive o que permite seu acesso por intermédio de dispositivos móveis.
\end{abstract}

Palavras-chave: Aprendizagem Ubíqua. Ambiente Virtual de Aprendizagem. Computação Ciente de Contexto.

\begin{abstract}
This paper presents a computational tool that integrates the concepts of ubiquitous learning in order to contribute to the process of teaching and learning. In this sense, it describes the development of a conversational agent, integrated into the virtual environment learning Moodle, which aims to provide support to the teaching of the discipline of Software Engineering. The agente entitled Ubibot, it is able to identify the student's context and answer the questions of the same. The contexts are represented by the level of performance and level student knowledge. Still the Ubibot uses web responsive technology which allows access through mobile devices.
\end{abstract}

Keywords: Ubiquitous Learning, Virtual Learning Environments. ContextAware Computing.

\section{Introdução}

A evolução das tecnologias computacionais permite a criação e atualização de sistemas voltados ao ensino eletrônico. Neste contexto, emerge a computação ubíqua e suas tecnologias, como recursos promissores que evidenciam tornar os ambientes virtuais de aprendizagem altamente dinâmicos. A computação ubíqua em consonância aos ambientes de ensino permite o surgimento do paradigma denominado aprendizagem ubíqua - ubiquitous learning (NETO; SALES, 2015). Desse modo, a aprendizagem ubíqua elabora um cenário em que os ambientes se adaptam em função da mobilidade e contexto do estudante (QUINTA; LUCENA, 2012). 
Aprendizagem ubíqua é um termo que unifica os conceitos de computação ubíqua e educação, em que objetiva-se uma aprendizagem transparente. Nesse sentido, a educação do estudante é realizada considerando as características do mesmo, ou seja, o ambiente de aprendizagem cria um perfil para cada aluno e ao estar neste ambiente, o mesmo terá um estudo direcionado às suas habilidades, conhecimentos e competências, independente de lugar e dispositivo (BARBOSA et al. 2014).

$\mathrm{Na}$ literatura é possível encontrar diversos estudos os quais descrevem sobre métodos utilizados para confeccionar ambientes de ensino ubíquo. Cita-se como exemplos os trabalhos de Mozzaquatro et al. (2010) e Possobom et al. (2014).

Mozzaquatro et al. (2010) realizaram uma pesquisa em que o ambiente virtual de aprendizagem Mobile Learning Engine Moodle se adapta ao estilo cognitivo do estudante, através de um framework denominado SEDECA, garantindo uma aprendizagem direcionada às preferências dos estudantes por materiais didáticos e o acesso via dispositivo móvel. Neste sentido, Mozzaquatro et al. (2010) consideraram o estilo cognitivo do estudante como uma informação que poderia ser utilizada para direcionar o ambiente aos diversos perfis do aprendiz.

Possobom et al. (2014) criaram uma extensão para o ambiente Moodle. Essa extensão recebeu a nomenclatura de SISTEX e possui a capacidade de identificar o nível de conhecimento do estudante na disciplina de Redes de Computadores. Nesse sentido, os autores consideraram o nível de conhecimento dos estudantes, como um contexto a ser identificado, para criar um ambiente que apresenta o conteúdo direcionado conforme o nível de conhecimento detectado.

Em ambientes de aprendizagem ubíqua, é necessário suportar a identificação de contexto (e.g. nível de conhecimento, estilo cognitivo, informações tecnológicas) e também a adaptação em diferentes tipos de dispositivos (e.g. smartphone, tablet, TV Digital, desktop). Nesse contexto, o objetivo da aprendizagem ubíqua é a projeção de sistemas interativos, dinâmicos e adaptativos.

No que se refere à customização de ambientes virtuais de aprendizagem para torná-los mais interativos, há estudos que descrevem sobre o uso de agentes conversacionais. Os agentes conversacionais são ferramentas tecnológicas que permitem simular conversas com seres humanos em linguagem natural. Eles são utilizados como entidades para instruir o estudante durante o aprendizado de conteúdos específicos (BADA; MENDEZ, 2012).

Agentes conversacionais objetivam compreender as necessidades dos usuários, utilizando recursos textuais ou sonoros para manter uma conversa (KONZEN et al. 2011). Desse modo, um agente possui uma base de conhecimento constituída por perguntas e respostas sobre um assunto, o qual o agente possui domínio. Por meio de perguntas realizadas pelo estudante, o agente consegue manter uma conversa com o usuário, representando um tutor pedagógico que provê suporte ao ensino de conteúdos (HERPICH et al. 2016).

$\mathrm{Na}$ literatura é possível encontrar diversos estudos que utilizam agentes conversacionais em ambientes virtuais de aprendizagem, tais como: Santos (2009) e Fossatti et al. (2011). Os agentes são inseridos em cursos ministrados no ambiente virtual, tendo como meta orientar o estudante durante as dúvidas na resolução de atividades (SILVA et al. 2012), podem reduzir a carga cognitiva do material didático 
disponibilizado pelo docente que ministra a disciplina (SANTOS, 2009) e apoiar o processo de ensino-aprendizagem de maneira inteligente (HERPICH et al. 2016).

Buscando desenvolver tecnologias que permitem o surgimento de ambientes virtuais de aprendizagem mais dinâmicos, este artigo apresenta uma pesquisa em andamento, que consiste no desenvolvimento de um agente conversacional o qual oferece suporte ao ensino de Engenharia de Software considerando o contexto do estudante. $\mathrm{O}$ agente é sensível ao contexto e se adapta a diferentes tipos de dispositivos computacionais, para criar um nível de ubiquidade aos usuários. Portanto, o agente é capaz de orientar o aluno considerando o contexto identificado e o dispositivo utilizado para acesso.

O agente conversacional denomina-se Ubibot, uma aglutinação entre as palavras Chatterbots e Ubiquitous Learning. Ele é capaz de identificar o nível de conhecimento do estudante matriculado na disciplina de Engenharia de Software e também o seu desempenho. Nesta pesquisa, o nível de conhecimento e o nível de desempenho são considerados como contextos a serem identificados para adaptar as orientações que o agente realiza ao estudante.

Este artigo está estruturado da seguinte forma: a segunda seção apresenta os trabalhos correlatos a esta pesquisa; a terceira seção contextualiza o agente conversacional desenvolvido; por fim, na quarta seção são evidenciadas algumas considerações e também são descritos trabalhos futuros.

\section{Trabalhos correlacionados}

Diversos estudos no contexto de agentes conversacionais inseridos em ambientes virtuais de aprendizagem têm sido realizados. O objetivo é criar ambientes dinâmicos que possam colaborar com o processo de ensino-aprendizagem. Desse modo, para o desenvolvimento do Ubibot algumas pesquisas foram analisadas, a fim de identificar possíveis recursos que podem ser incorporados no agente, bem como as tecnologias envolvidas na confecção dos mesmos.

Para demonstrar quais tecnologias estão presentes neste trabalho e fazer uma relação com as utilizadas em estudos anteriores a esta pesquisa, foram escolhidos cinco trabalhos: Jaques e Vicari (2005), Santos (2009), Borin (2010), Fossati et al. (2011) e Herpich et al. (2016).

No estudo de Jaques e Vicari (2005) é relatado o desenvolvimento de um agente pedagógico animado. O agente denominado PAT é capaz de ajudar o estudante durante a sua interação no ambiente virtual de aprendizagem Multi-Agent Architecture for an Collaborative Educational System (MACES). Para isso, PAT parte da premissa de auxiliar o estudante quando ele necessita de ajuda, garantindo o encorajamento do mesmo durante as dificuldades que são evidenciadas no processo de aprendizagem. PAT possui sensores virtuais que capturam o comportamento do estudante e a partir dessa identificação, o agente é capaz de expressar comportamentos emotivos a fim de motivar e encorajar o aluno. Para expressar emoções, Jaques e Vicari (2005) codificaram o agente considerando a abordagem Belief-Desire-Intention (BDI).

Santos (2009) criou em sua tese um agente conversacional que objetiva ajudar o estudante na resolução de atividades. Ele foi inserido no ambiente virtual de aprendizagem Moodle, tendo como meta, auxiliar o aluno quando o mesmo possuir 
dúvidas referentes ao conteúdo do curso de Softwares Educacionais. O agente desenvolvido possui a capacidade de interagir com os usuários por meio de linguagem natural, visto que Santos (2009) utilizou a linguagem Artificial Intelligence Markup Language (AIML) para implementar os diálogos. A base de conhecimento é composta por recursos de AIML que permitem tornar a conversa atrativa ao estudante. Para representar graficamente a aparência do agente em duas dimensões, foi utilizada a ferramenta OddCast $^{1}$. Neste estudo, Santos (2009) evidenciou que um agente conversacional pode influenciar de forma positiva no aprendizado dos alunos.

Borin (2010) desenvolveu o agente pedagógico emocional tridimensional Doris, que permite a comunicação por intermédio de linguagem natural. $\mathrm{O}$ agente foi integrado em ambiente virtual de aprendizagem tendo como intuito oferecer suporte ao estudante. Para ajudar o aluno, o agente possui a capacidade de expressar emoções tais como: tristeza, alegria, surpresa, indignação, expectativa, atenção e dúvida em suas feições. Doris possui sensores virtuais capazes de identificar as páginas que o estudante visualiza. Desse modo, ele percebe se o aluno não visualizou um conteúdo importante e emite avisos e questionamentos ao estudante, fazendo sempre o uso de táticas emotivas.

Fossatti et al. (2011) descrevem sobre o desenvolvimento de um agente conversacional denominado AGEbot. O agente tem como meta responder dúvidas referentes ao assunto de epilepsia. AGEbot foi implementado na linguagem AIML, apoiado pela plataforma Pandorabots. Nesse sentido, o agente é capaz de interpretar as perguntas realizadas em linguagem natural e respondê-las. Além disso, os autores utilizaram uma estratégia de aprendizagem baseada em reforço, para inserir novos conhecimentos e habilidades ao agente.

No trabalho de Herpich et al. (2016), os autores relatam sobre a implementação do agente ELAI. Esse agente é agregado em um Mundo Virtual tridimensional a fim de ajudar o estudante matriculado na disciplina de Redes de Computadores. O ELAI possui a capacidade de adaptar-se conforme o estilo cognitivo e nível de conhecimento do usuário. A adaptação ocorre por meio de módulos SEDECA e SISTEX integrados ao ambiente tridimensional, garantindo ao ELAI uma atuação orientada ao contexto detectado. $\mathrm{O}$ agente também possui a capacidade de interagir com os usuários por meio de linguagem natural, pois foi desenvolvido com auxílio da plataforma Pandorabots. Ressalta-se que esse agente auxilia o estudante considerando o contexto, ou seja, ao fazer login no Mundo Virtual o estudante é direcionado a um ambiente que é constituído por materiais voltados ao estilo cognitivo do aluno e apresenta um agente que possui a base de conhecimento direcionada ao contexto de nível de conhecimento.

Objetivando evidenciar as principais tecnologias envolvidas nos agentes descritos nessa seção, foi elaborada uma tabela. A Tabela 1 evidencia alguns recursos utilizados e permite dar uma noção de quais tecnologias podem ser utilizadas para implementar o agente Ubibot.

O termo SIM representa que a tecnologia ou recurso é utilizado no agente e o termo NÃO demonstra que a tecnologia não faz parte da arquitetura do agente.

\footnotetext{
${ }^{1}$ www.oddcast.com
} 
Tabela 1 - Tecnologias e recursos dos agentes pesquisados

\begin{tabular}{|c|c|c|c|c|c|}
\hline$\underbrace{\text { Agente }}_{\text {Recurso/Tecnologia }}$ & PAT & $\begin{array}{l}\text { Agente de } \\
\text { Santos } \\
(2009)\end{array}$ & Dóris & AGEbot & ELAI \\
\hline Abordagem BDI & SIM & NÃO & NÃO & NÃO & NÃO \\
\hline AIML & NÃO & SIM & NÃO & SIM & SIM \\
\hline Aprendizado por reforço & NÃO & NÃO & NÃO & SIM & NÃO \\
\hline AVA & SIM & SIM & SIM & SIM & NÃO \\
\hline Comportamento Emotivo & SIM & NÃO & SIM & NÃO & NÃO \\
\hline Estilo Cognitivo & NÃO & NÃO & NÃO & NÃO & SIM \\
\hline Modelagem em 3D & NÃO & NÃO & SIM & NÃO & SIM \\
\hline Mundo Virtual & NÃO & NÃO & NÃO & NÃO & SIM \\
\hline Nível de Conhecimento & NÃO & NÃO & NÃO & NÃO & SIM \\
\hline OddCast & NÃO & SIM & NÃO & NÃO & NÃO \\
\hline Pandorabots & NÃO & NÃO & NÃO & SIM & SIM \\
\hline Sensor Virtual & SIM & NÃO & SIM & NÃO & SIM \\
\hline
\end{tabular}

A partir da Tabela 1, foi possível definir alguns recursos utilizados posteriormente no Ubibot. Nesse sentido, o agente Ubibot é desenvolvido na linguagem AIML com auxílio da plataforma Program $O^{2}$, possui uma representação gráfica de duas dimensões, foi inserido no ambiente virtual de aprendizagem Moodle, não apresenta comportamento emotivo, possui sensores virtuais para identificar o nível de conhecimento e o nível de desempenho do estudante, não considera o contexto cognitivo do aluno (estilo cognitivo) e possuirá um feedback orientado ao contexto do usuário. Destaca-se o acesso independente de dispositivo, pois o agente é integrado ao ambiente Moodle que apresenta um tema constituído pelo framework Bootstrap.

Na próxima seção é apresentado o agente conversacional Ubibot. Na mesma serão descritas as funcionalidades do agente e também o processo de desenvolvimento.

\section{Agente Ubibot}

Falkembach (2003) descreve que os ambientes virtuais de aprendizagem podem mapear o nível de conhecimento prévio do estudante, sobre determinado conteúdo, antes de apresentar o material de uma disciplina ao aprendiz. Normalmente, os alunos não possuem o mesmo nível de conhecimento ao iniciar seu aprendizado em uma disciplina. Ao detectar o conhecimento prévio do aluno é possível adequar o conteúdo programático da disciplina as suas necessidades.

Os autores Possobom et al. (2014), utilizam a premissa descrita no parágrafo anterior para criar um sistema capaz de identificar o nível de conhecimento do estudante de Redes de Computadores e a partir dessa detecção, o sistema adapta o ambiente Moodle ao contexto. Para identificar o nível de conhecimento Possobom et al. (2014) elaboraram um questionário composto por dez questões que abrangem o conteúdo programático da disciplina. Os autores elaboram uma regra de condição para identificar o nível de conhecimento: se o usuário acertar entre zero até quatro questões, seu nível de conhecimento será básico; se o usuário acertar mais que quatro e no máximo sete, seu nível de conhecimento será intermediário; se o usuário acertar mais do que sete questões, seu nível de conhecimento será avançado. A partir do estudo realizado por

\footnotetext{
${ }^{2}$ É uma máquina de inferência semelhante ao Pandorabots, que permite a interpretação de códigos AIML. Disponível em <http://www.program-o.com/>
} 
Possobom et al. (2014), os autores Herpich et al. (2016) fizeram uso do SISTEX como um módulo para o agente ELAI.

Ao considerar a necessidade da criação de um mecanismo para identificar o nível de conhecimento de estudantes de Engenharia de Software, implementou-se um módulo composto por dez questões de maneira semelhante ao sistema desenvolvido por Possobom et al. (2014). As questões foram retiradas do Exame Nacional de Desempenho dos Estudantes (ENADE) e também do Exame Nacional para Ingresso na Pós-Graduação em Computação (POSCOMP). O módulo é capaz de identificar o nível de conhecimento de estudantes de Engenharia de Software.

A Figura 1 apresenta a interface inicial do modelo capaz de identificar o nível de conhecimento de estudante. Portanto, quando o usuário acessa o ambiente Moodle pela primeira vez, o sistema solicita ao estudante o preenchimento de um questionário. Ressalta-se que nessa pesquisa, a ideologia de Possobom et al. (2014) é mantida como um meio de identificação de um tipo de contexto.

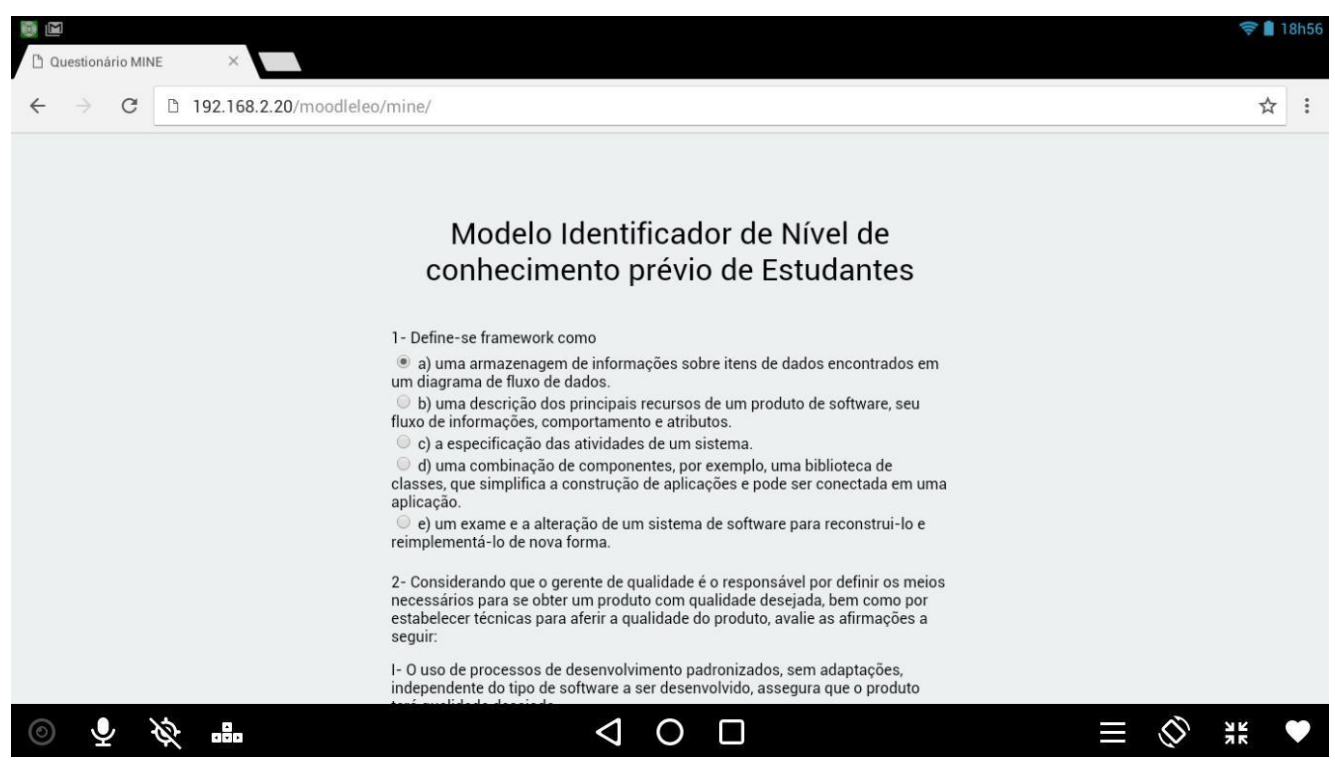

Figura 1 - Modelo Identificador de Nível de Conhecimento de Estudantes

Este estudo parte da ideologia que o estudante evoluirá no decorrer da disciplina, e não possuirá o mesmo conhecimento durante a interação com a mesma, pois aprenderá novos conceitos. Por esse motivo, o nível de conhecimento é considerado até o momento em que o ambiente não possui atividades avaliadas. Quando existirem atividades avaliadas o Ubibot começará a considerar o desempenho do aluno na disciplina ao apresentar as suas orientações, substituindo o contex to com base no nível de conhecimento.

Dias et al. (2009) classificam o desempenho do estudante em três categorias: baixo desempenho; bom desempenho; excelente desempenho. Para realizar essa classificação os autores consideram a média geral do aluno. Na Tabela 2 é possível evidenciar o fator delimitador que classifica o desempenho do estudante.

Tabela 2 - Identificação do Nível de Desempenho do Estudante

\begin{tabular}{|r|l|}
\hline NÍVEL DE DESEMPENHO & FATOR DELIMITADOR \\
\hline Baixo desempenho & $0 \leq$ Media_geral $<70 \%$ \\
\hline Bom desempenho & $70 \% \leq$ Media_geral $\leq 80 \%$ \\
\hline Excelente desempenho & $80 \%<$ Media_geral $\leq 100 \%$ \\
\hline
\end{tabular}


$\mathrm{Na}$ pesquisa apresentada o nível de desempenho é considerado como um contexto do estudante, porque é uma informação relevante que pode ser utilizada. Basicamente, os funcionamentos dos módulos que identificam os contextos do aluno ocorrem da seguinte forma: Primeiramente, quando o aluno acessa o ambiente Moodle, o agente executa um algoritmo para verificar se existe alguma atividade avaliada no ambiente, caso exista, o ambiente identifica o nível de desempenho do aluno, considerando o fator delimitador exposto na Tabela 2 e apresenta o agente Ubibot. Caso não exista atividade avaliada, o ambiente verifica se o nível do conhecimento do estudante já foi detectado, em caso positivo, é apresentado o agente Ubibot, em caso negativo, o questionário é carregado, pois o agente busca detectar o nível de conhecimento do estudante.

Toda vez que o estudante de Engenharia de Software acessa o ambiente, o Ubibot verifica o seu contexto. A Figura 2 apresenta o caso de uso geral, nele é possível observar que o estudante e o agente Ubibot são atores e cada um deles possui diversos casos de uso (funções).

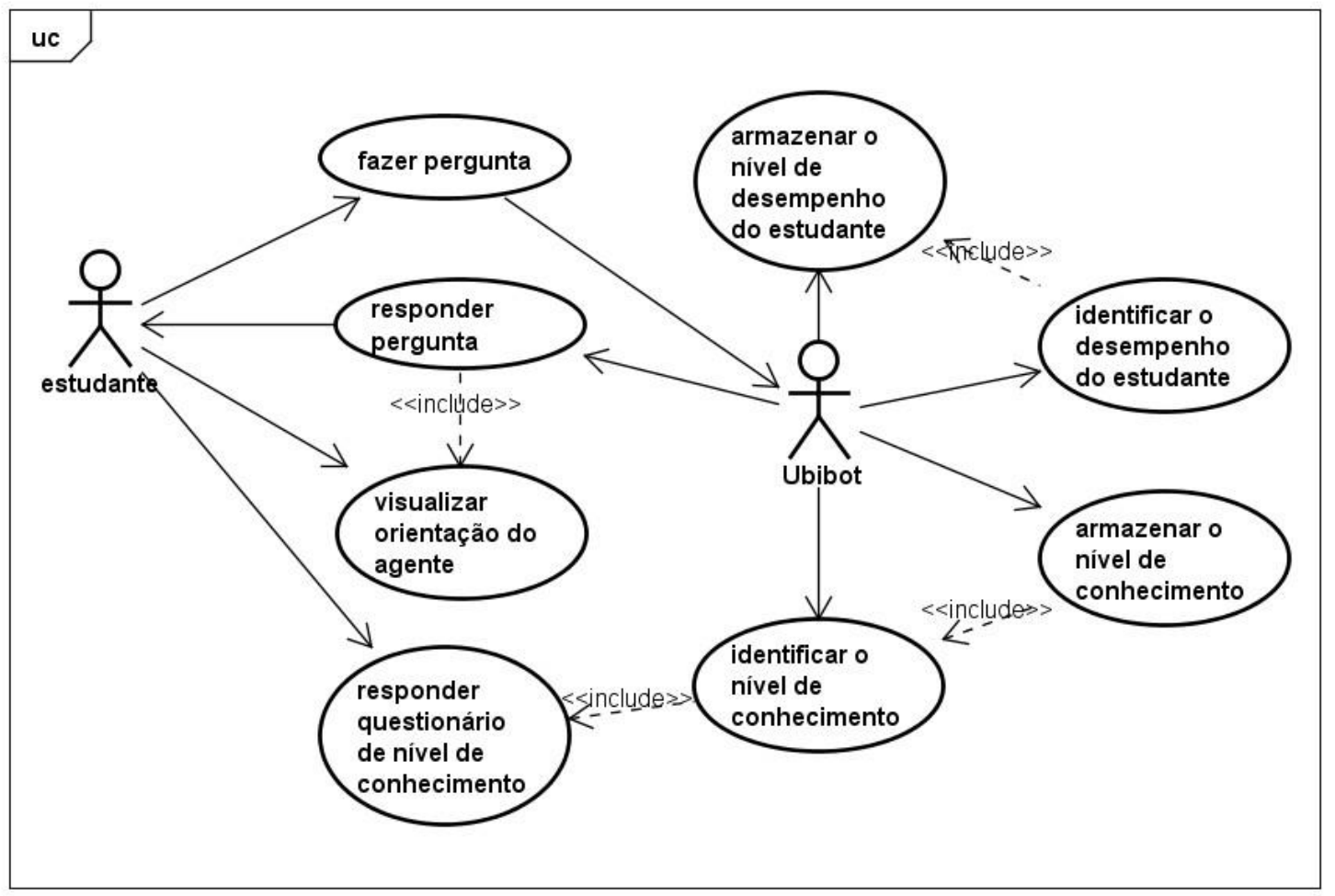

Figura 2 - Casos de uso geral do Ubibot

A construção do Ubibot considerou os elementos expostos na Tabela 1. Desse modo, foram utilizados recursos tecnológicos como o Program $O$ para armazenar a base de conhecimento e a ferramenta online Voki para elaborar uma representação gráfica do agente Ubibot. A Figura 3 apresenta o Ubibot sendo acessado por um dispositivo móvel.

Por tratar-se de um agente conversacional, optou-se por utilizar a linguagem de marcação AIML. Com essa linguagem, o agente conseguirá interpretar as perguntas realizadas pelo estudante. Considerados os contextos que o agente suporta (nível de conhecimento e nível de desempenho), foram criadas três bases de conhecimentos para o agente Ubibot. Cada uma delas voltada a um contexto, sendo elas: base um - 
conhecimento básico ou baixo desempenho; base dois - conhecimento intermediário ou bom desempenho; base três - conhecimento avançado ou excelente desempenho.

Foi necessário inserir duas tabelas no ambiente Moodle, sendo elas: $m d l \_m i n e$ e mdl_mide. A tabela $m d l \_m i n e$ é responsável por armazenar os dados de nível de conhecimento do estudante e a tabela mdl_mide é responsável por armazenar os dados de nível de desempenho. Além disso, consultaram-se dados em diversas tabelas originais do ambiente Moodle, a fim de coletar informações sobre o estudante, para posteriormente o agente apresentar seu feedback orientado ao contexto. As tabelas originais do Moodle que foram utilizadas são: mdl_grade_items, mdl_grade_grades, mdl_enrol, $m d l \_u s e r \_e n r o l m e n t s$, mdl_context, $m d l \_r o l e \_a s s i g n m e n t s$, entre outras.

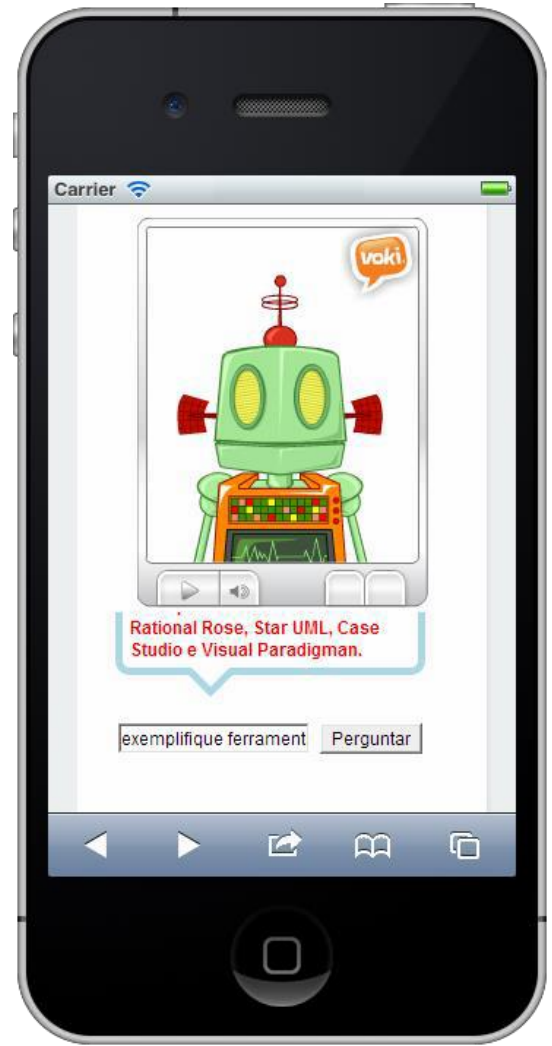

Figura 3 - Ubibot exemplificando ferramentas $\mathrm{CASE}^{3}$

Para o agente personalizado aparecer ao estudante, foram criados três blocos em HyperText Markup Language (HTML) no Moodle, um para cada contexto. Também foram criados três novos papeis para os usuários, garantindo um para cada tipo de perfil. Posteriormente em cada bloco foram inseridos um agente com sua base de conhecimento específica (e.g. base um, base dois, base três). Por fim, os blocos receberam configurações no que se refere à visualização, assim, o primeiro bloco será visível apenas aos estudantes com nível de conhecimento básico ou baixo nível de desempenho, o segundo bloco será visível apenas aos estudantes que possuem nível de conhecimento intermediário ou bom nível de desempenho, por fim, o terceiro bloco será visível apenas aos estudantes que possuem nível de conhecimento avançado.

\footnotetext{
${ }^{3}$ Computer-Aided Software Engineering
} 


\section{Considerações Finais}

Este artigo buscou apresentar um agente conversacional que provê suporte ao ensino de Engenharia de Software. O agente é sensível ao contexto do nível de conhecimento e nível de desempenho do estudante e também se adapta à interface de diferentes dispositivos móveis, com auxílio do tema web responsive Bootstrap.

Identificou-se que o Ubibot consegue apresentar as respostas personalizadas aos estudantes, de maneira semelhante ao agente ELAI. Observou-se que o avatar o qual representa o agente não consegue ser executado em dispositivos móveis que não possuem o Flash Player. Trata-se de uma limitação do Ubibot. Como a maioria dos dispositivos não possuem o Flash Player, evidencia-se alterar a ferramenta para representar graficamente o Ubibot, optando-se por uma representação gráfica desenvolvida em HTML5.

O método de aprendizagem utilizado por Fossatti et al. (2011), está sendo estudado, para posteriormente ser implementado como um novo recurso ao Ubibot. Portanto, o Ubibot ainda não possui característica de agente baseado em aprendizagem de máquina.

Em pesquisas futuras objetiva-se utilizar outro recurso para criar o avatar de interface. Também será realizada uma validação do agente Ubibot com estudantes da disciplina de Engenharia de Software. É possível que durante a avaliação sejam utilizados métodos semelhantes aos estudos de Herpich et al. (2016) e Fossatti et al. (2011). Futuramente visa-se ampliar a base de conhecimento do agente, a fim de criar recursos que ofereçam suporte ao diálogo mais consistes e criativos, no que se refere ao conteúdo da disciplina.

\section{Referências Bibliográficas}

BADA, E, M.; MENEZES, C. S. Uma proposta para extração de perguntas e respostas. In: XVII Congreso Internacional de Informática Educativa (TISE), Chile, 2012.

BARBOSA, J. L.; BARBOSA, D. N.; RIGO, S. J., OLIVEIRA; J. M. DE; RABELLO Jr., S. A. Integrating Collaborative and Decentralized Models to Support Ubiquitous Learning. In: International Journal of Information and Communication Technology Education (IJICTE), 10(3), 77-86, 2014. DOI: 10.4018/ijicte.2014070106

BORIN, M. O uso de Ambientes Virtuais de Aprendizagem com Agentes Pedagógicos Emocionais para capacitação em organizações. Programa de PósGraduação em Sistemas e Processos Industriais/ Universidade de Santa Cruz do Sul, 2010. 120p. Dissertação de Mestrado.

DIAS, A. F. M.; CERQUEIRA, G. S.; LINS, L. N. Fatores determinantes da retenção estudantil em um curso de graduação em engenharia de produção. In: XXXVII Congresso Brasileiro de Educação em Engenharia, 2009.

FALKEMBACH, G. A. M. Uma experiência de resolução de problemas através da estratégia ascendente - Ambiente de Aprendizagem Adaptado para Algoritmos (A4). Centro de Novas Tecnologias na Educação/ Universidade Federal do Rio Grande do Sul, 2003. 190p. Tese de Doutorado.

FOSSATTI, M. ; RABELLO, R. S.; DE MARCHI, A. C. B. AGEbot: Um chatterbot em AIML voltado para responder questões sobre Epilepsia. In: XI Workshop de 
Informática Médica - XXXI Congresso da Sociedade Brasileira de Computação, p. 1876-1882, 2011.

HERPICH, F.; VOSS, G. B.; NUNES, F. B.; MEDINA, R. D. Three-Dimensional Virtual Environment and NPC: A Perspective about Intelligent Agents Ubiquitous. In: Francisco Milton Mendes Neto; Rafael de Souza; Alex Sandro Gomes. (Org.). Handbook of Research on 3-D Virtual Environments and Hypermedia for Ubiquitous Learning. 1ed.: IGI Global, 2016, v. 21, p. 510-536. DOI: 10.4018/978-15225-0125-1.ch021

JAQUES, P. A.; VICCARI, R M. PAT: um agente pedagógico animado para interagir efetivamente com o aluno. Revista Novas Tecnologias na Educação (RENOTE), v. 3, (1), 2005.

KONZEN, A. A.; OLIVEIRA, O. L, O.; KIST, L. S.; ANJOS, A. R.; MORAES, L.; FREITAS, C. I. L.; MÜLLER, D. N.; AXT, M. Mega Vitta - agente conversacional aplicado ao jogo educacional Città. In: XXII Simpósio Brasileiro de Informática na Educação, 2011. DOI: http://dx.doi.org/10.5753/cbie.sbie.2011.\%25p

MOZZAQUATRO, P. M.; FRANCISCATO, F. T.; RIBEIRO, P. S.; MEDINA, R. D. Ambiente Virtual de Aprendizagem Móvel adaptado aos diferentes estilos cognitivos utilizando Hipermídia Adaptativa. In: XVI Workshop Sobre Informática na Escola - , 2010. DOI: http://dx.doi.org/10.5753/cbie.wie.2010.1255-1264

NETO, F. M. M.; SALES, A. F. A. A Recommendation System for Ubiquitous Learning in the Context of Formal and Informal Education. In: IEEE Latin America Transactions, v. 13, n.4, 1061-1067, 2015. DOI: 10.1109/TLA.2015.7106358

POSSOBOM, C.C.; MÜHLBEIER, A. R. K.; NUNES, F. B.; CARVALHO, A.; GOMES, R. B.; MEDINA, R. D. Uma Aplicação Dinâmica para Detectar o Nível de Conhecimento do Aluno. In: XXIII Ciclo de Palestras Novas Tecnologias na Educação, 2014.

QUINTA, M. R.; LUCENA, F. N. Adaptação de material didático para u-learning: sistema Odin. Revista Brasileira de Informática na Educação, v. 20, n.1, 2012. DOI: http://dx.doi.org/10.5753/RBIE.2012.20.1.75

SANTOS, L. M. A. A Inserção de um Agente Conversacional Animado em um Ambiente Virtual de Aprendizagem a partir da Teoria da Carga Cognitiva. Centro Interdisciplinar de Novas Tecnologias na Educação/ Universidade Federal do Rio Grande do Sul, 2009. 125p. Tese de Doutorado.

SILVA, J. H.; COUTO, L. S.; ODAKURA, V. Aparência de um agente pedagógico animado para um ambiente virtual de aprendizagem. In: XXIII Simpósio Brasileiro de Informática na Educação, 2012. DOI: http://dx.doi.org/10.5753/cbie.sbie.2012.\%25p 